\title{
Renal Tuberculosis Presenting as a Mass Lesion in a Two-year-old Girl Report of a rare case
}

"Avradip Santra, ${ }^{1}$ Falguni Mandi, ${ }^{*}$ Abhishek Bandyopadhyay ${ }^{2}$

$$
\text { أفراديب سنترا، فلاجوني مندي، أبهيشك بـاندى أويـاديا }
$$

ABSTRACT: Genitourinary tuberculosis usually occurs in young adults and the middle-aged and is very uncommon in the paediatric population. It generally presents with haematuria, pyuria, irritative voiding symptoms and flank pain; presentation as a renal mass is highly unusual. We report a two-year-old girl who was referred to the Nil Ratan Sircar Medical College, Kolkata, India, in June 2014 with abdominal pain. Subsequent radiological investigations revealed a left renal hypoechoic mass lesion. A left nephroureterectomy was performed on suspicion of a Wilms' tumour. Histopathology indicated an epithelioid granuloma with lymphocytic infiltration, suggestive of a tubercular aetiology. A Mantoux tuberculin skin test was positive; however, there was no evidence of tuberculosis detected elsewhere in the body and the source of the infection could not be identified. A diagnosis of renal tuberculosis was made and the child was treated with antitubercular drugs. The patient was asymptomatic at a six-month follow-up.

Keywords: Renal Tuberculosis; Urogenital Tuberculosis; Abdominal Pain; Granuloma; Case Report; India.

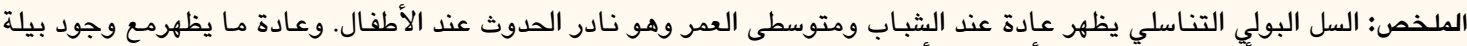

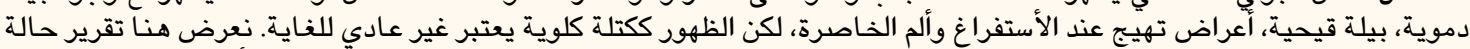

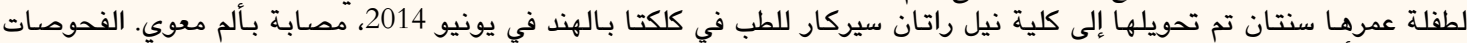

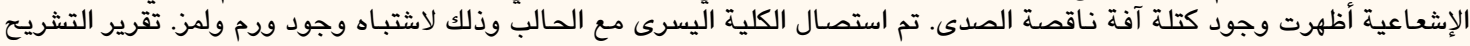

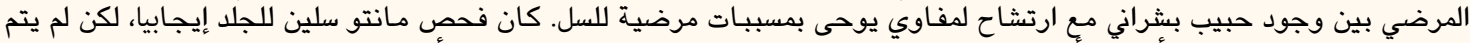

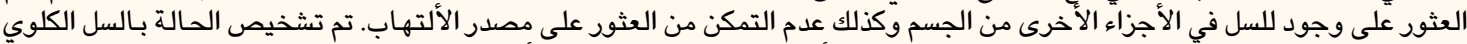

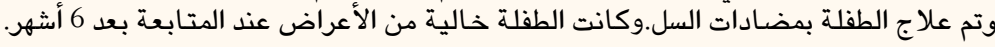

$$
\text { مفتاح الكلمات: السل الكلوي؛ السل البولي التناسلي؛ ألم البطن؛ حبي؛ تقرير حالة؛ الهند. }
$$

A CCORDing TO THE WORLD HeALTH Organization, the annual tuberculosis (TB) burden in India was 2.2 million in 2012; this figure constitutes one quarter of global TB cases per year. ${ }^{1}$ Genitourinary TB is a common form of extrapulmonary TB, accounting for $27 \%$ (range: 14-41\%) of all cases of extrapulmonary $\mathrm{TB}$ in developed countries. ${ }^{2}$ Usually, genitourinary TB is a complication in $3-4 \%$ of pulmonary TB cases. ${ }^{3}$ Active genitourinary $\mathrm{TB}$ generally occurs between five and 15 years after a primary pulmonary infection with Mycobacterium tuberculosis. ${ }^{4}$ Despite its status as the most common form of genitourinary TB, renal TB is very rare in the paediatric population. ${ }^{5}$

Genitourinary TB mostly presents with irritative voiding symptoms, haematuria and flank pain. ${ }^{6}$ Presentation as a mass lesion is extremely rare and few cases have been published in the literature to date. $^{7-10}$ This report describes a two-year-old child who presented with a renal mass and was diagnosed with renal TB via a post-nephroureterectomy histopathological examination.

\section{Case Report}

A two-year-old girl presented to a private health clinic in Kolkata, India, with non-specific abdominal pain of three months' duration. As she did not improve with symptomatic management and anthelmintic therapy, she was referred to the Nil Ratan Sircar Medical College, Kolkata, India, in June 2014 for further evaluation. There was no history of fever, vomiting, altered bowel habits, abdominal distension or changes in urinary frequency or colour over the preceding three months. In addition, no appetite or weight loss had been observed by the parents. The child 


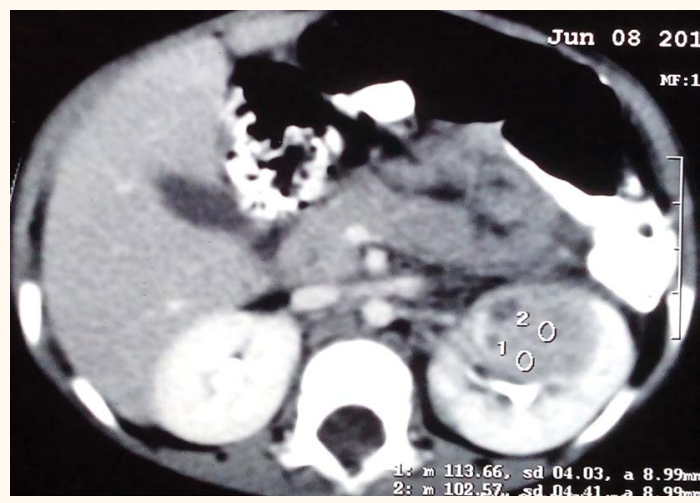

Figure 1: Contrast-enhanced abdominal computed tomography scan showing a well-defined hypodense mass lesion in the left kidney of a two-year-old girl with renal tuberculosis.

had been delivered at full term at home and was not immunised. She had no prior significant illnesses and her developmental history was normal. There were no signs of pallor, icterus, palpable lymphadenopathy or malnutrition. An abdominal examination revealed no palpable organomegaly or tenderness. The results of routine biochemical tests and a complete haemogram were within normal ranges and the child was negative for human immunodeficiency virus infection.

Ultrasonography of the abdomen revealed a hypoechoic mass lesion in the left kidney along with mild hepatomegaly without any abdominal lymphadenopathy, splenomegaly or ascites. Subsequently, a computed tomography scan of the abdomen was performed with intravenous and oral contrast. A well-defined hypodense space-occupying lesion ( $28 \times 23 \times 32 \mathrm{~mm})$ with heterogeneous enhancement was observed at the mid-pole of the left kidney compressing the left renal pelvis [Figure 1]. No other abnormalities were observed. The liver was mildly enlarged, but otherwise normal in terms of position, shape and contour. The intrahepatic biliary radicles were also normal. These findings were suggestive of a Wilms' tumour and a left nephroureterectomy was deemed necessary.

Following the surgery, a histopathological examination of the excised mass revealed granulomatous interstitial nephritis with central areas of caseous necrosis. An epithelioid granuloma was observed along with Langhans giant cells and dense lymphoplasmacytic infiltrate with lymphoid follicle formation. The granuloma was found within the perinephric fat [Figure 2]; however no evidence of acid-fast bacilli (AFB) was found with Ziehl-Neelsen staining. A Mantoux tuberculin skin test using two tuberculin units of purified protein derivative RT-23 was positive with an induration of $12 \mathrm{~mm}$. The patient underwent further evaluations for congenital TB;

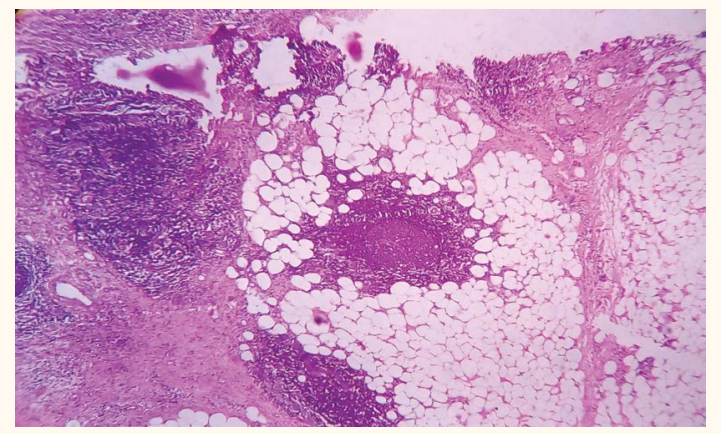

Figure 2: Haematoxylin and eosin stain at x10 magnification showing a caseating epithelioid granuloma and lymphoid follicle within the perinephric fat of a two-year-old girl with renal tuberculosis.

however, her chest X-ray was normal and a gastric lavage was negative for $M$. tuberculosis. Morning urine samples taken on three consecutive days were AFB-negative and a urine culture was also negative for $M$. tuberculosis. There was no evidence of active TB on chest X-rays or AFB in sputum examinations for any of the patient's family members. An endometrial biopsy was requested from the child's mother; however, this was not performed as she was completely asymptomatic and did not give consent for the investigation.

The patient was diagnosed with renal TB based on the findings of the renal histopathology and ancillary investigations. She subsequently underwent two months of intensive phase antitubercular therapy with isoniazid, rifampicin, pyrazinamide and ethambutol, followed by four months of continuation phase therapy with isoniazid and rifampicin, as per the guidelines of the Revised National Tuberculosis Control Programme (RNTCP). ${ }^{11}$ She improved symptomatically and the abdominal pain subsided within one month of beginning the treatment. By the end of the six-month antitubercular therapy, she was completely asymptomatic. At the time of writing, the patient was being followed up at regular intervals.

\section{Discussion}

Genitourinary TB is most commonly encountered between the second and fourth decade of life. ${ }^{3,6}$ This form of TB represents less than 5\% of paediatric extrapulmonary cases worldwide and is very infrequently reported in the literature. ${ }^{12,13}$ Chattopadhyay et al. observed only nine cases of paediatric genitourinary TB over a span of seven years among patients aged 5-12 years old. ${ }^{14}$ A case of congenital renal TB was reported in a 34-day-old infant presenting with exsanguinating haematuria. ${ }^{13}$ Another report described miliary TB with focal 
renal involvement in a five-month old male infant. ${ }^{15}$ Commonly, patients with renal TB present with dysuria, haematuria, sterile pyuria, flank pain, recurrent urinary tract infections and constitutional symptoms. ${ }^{6,13}$ However, the radiographic appearance of the condition is variable and depends on the stage of infection. ${ }^{16}$ An enhancing renal mass is a rare presentation; in such cases, renal cell carcinomas, renal metastasis, lymphomas or abscesses are usually considered in the differential diagnosis. ${ }^{17}$ Similarly to the present case, Dhua et al. noted a well-defined hypodense renal mass in a case of renal TB. ${ }^{13}$ Kumar et al. reported a pseudotumoural manifestation of genitourinary TB in a 34-year-old woman, which was initially suspected to be a renal cell carcinoma.? A solid tubercular large mass mimicking a tumour in a horseshoe kidney has also been described in the literature. $^{8}$

Haematogenous dissemination from an active TB infection site usually results in the formation of metastatic tubercles in the kidney which either heal spontaneously or as a result of antitubercular therapy. Renal lesions may sometimes progress during primary infection or enlarge after several years. ${ }^{3}$ In the current case, the route of infection could not be ascertained. There was no evidence of active TB elsewhere in the body or among family members. Additionally, the case did not meet the revised criteria for a diagnosis of congenital TB as described by Cantwell et al. ${ }^{18}$ Nevertheless, it is often very difficult to differentiate congenital TB from acquired neonatal TB when the baby is infected after birth. Unfortunately, a maternal endometrial biopsy could not be performed to rule out this possible source of infection.

Renal TB can be cured without surgical intervention; however, this requires timely diagnosis and a high degree of clinical suspicion. ${ }^{6}$ Unfortunately, the tubercular aetiology of the tumour-like mass in the current case was not suspected preoperatively and early radical surgery was performed. Fine-needle aspiration cytology (FNAC) is a recommended diagnostic procedure to avoid surgical intervention and retain the affected kidney. ${ }^{7}$ Additionally, the interferon gamma release assay (IGRA) test, which can detect $M$. tuberculosis infection anywhere in the body, is generally considered a more effective diagnostic tool than a conventional tuberculin skin test. ${ }^{19}$ However, this test was not carried out in the present case due to a lack of IGRA performance data in paediatric populations below five years of age. ${ }^{19}$ In addition, certain nontuberculous mycobacteria-including M. kansasii, M. szulgai and M. marinum-may produce false-positive results which could complicate the diagnostic procedure. ${ }^{19}$
Short-course chemotherapy is the standard pharmacological treatment for genitourinary $\mathrm{TB}$, as per the RNTCP guidelines. ${ }^{1,2}$ A six-month course of category I therapy can be highly efficacious; Singh et al. described positive urinary AFB, mycobacterial cultures or polymerase chain reaction tests in $86.4 \%$ of genitourinary TB cases which became negative within three months of antitubercular therapy. ${ }^{6}$ Nevertheless, surgery still plays an important role in genitourinary TB management despite the advent of modern antitubercular therapy. A partial nephrectomy is recommended for localised polar lesions containing calcification which fail to respond to six weeks of intensive chemotherapy or for areas of calcification which slowly increase in size and threaten to gradually destroy the entire kidney. ${ }^{6}$ Complete nephrectomies are indicated only for non-functioning tubercular kidneys with calcification or for cases of extensive disease involving the whole kidney occurring with other complications (e.g. hypertension or a coexistent renal cell carcinoma). ${ }^{20} \mathrm{~A}$ minimum of four weeks of antitubercular therapy is recommended before any major surgical intervention as it stabilises the lesion and allows greater accuracy during reconstructive surgery. ${ }^{6,21}$

\section{Conclusion}

Renal TB should be considered in the differential diagnosis of a paediatric renal mass. A high degree of suspicion is warranted, especially for patients from countries with a high prevalence of TB. Close family members should be thoroughly investigated in cases of suspected congenital TB to determine the source of infection. Preoperative diagnosis is recommended to avoid the need for radical surgery.

\section{References}

1. Directorate General of Health Services, Government of India. TB India 2014: Revised National TB Control Programme Annual status report. From: www.tbcindia.nic.in/showfile. php?lid=3142 Accessed: Sep 2015.

2. Eastwood JB, Corbishley CM, Grange JM. Tuberculosis and the kidney. J Am Soc Nephrol 2001; 12:1307-14.

3. HemalAK.Genitourinary tuberculosis. In:SharmaSK, Mohan A, Eds. Tuberculosis. 1st ed. New Delhi, India: Jaypee Brothers Medical Publishers, 2001. Pp. 325-37.

4. Wallgren A. The time-table of tuberculosis. Tubercle 1948; 29:245-51. doi: 10.1016/S0041-3879(48)80033-4

5. Hatzenbuehler LA, Starke JR. Tuberculosis (Mycobacterium tuberculosis). In: Kliegman RM, Stanton BM, St. Geme J, Schor NF, Eds. Nelson Textbook of Paediatrics. 20th ed. Philadelphia, Pennsylvania, USA: Elsevier, 2015. Pp. 1445-60.

6. Singh JP, Priyadarshi V, Kundu AK, Vijay MK, Bera MK, Pal DK. Genito-urinary tuberculosis revisited: 13 years' experience of a single centre. Indian J Tuberc 2013; 60:15-22. 
7. Kumar S, Shankaregowda SA, Choudhary GR, Singla K. Rare presentation of genitourinary tuberculosis masquerading as renal cell carcinoma: A histopathological surprise. J Clin Imaging Sci 2014; 4:26. doi: 10.4103/2156-7514.133259.

8. Gupta H, Kone K, Pandey S, Dorairajan LN, Kumar S. Tubercular mass mimicking a tumour in a horseshoe kidney: A unique presentation. Int Urol Nephrol 2004; 36:323-4. doi: 10.1007/s11255-004-0925-y.

9. Sood R, Mukhopadhyay S. Unusual imaging features in a case of renal tuberculosis presenting as fever of unknown origin. J Indian Acad Clin Med 2001; 2:87-90.

10. Dinolfo M. Renal tuberculosis presenting as renal mass. Proc UCLA Healthcare 2013; 17:1.

11. Verma R, Khanna P, Mehta B. Revised National Tuberculosis Control Program in India: The need to strengthen. Int J Prev Med 2013; 4:1-5.

12. Starke JR, Smith KC. Tuberculosis. In: Feigin RD, Cherry J, Demmler G, Kaplan S, Eds. Textbook of Pediatric Infectious Diseases. Philadelphia, Pennsylvania, USA: Saunders, 2003. Pp. 1337-79.

13. Dhua AK, Borkar N, Ghosh V, Aggarwal SK. Renal tuberculosis in infancy. I Indian Assoc Pediatr Surg 2011; 16:69-71. doi: 10.4103/0971-9261.78136.

14. Chattopadhyay A, Bhatnagar V, Agarwala S, Mitra DK. Genitourinary tuberculosis in pediatric surgical practice. J Pediatr Surg 1997; 32:1283-6. doi: 10.1016/S0022-3468 (97)90302-X.
15. Kay AW, McCarthy CA. Renal and miliary tuberculosis in an internationally adopted infant. Pediatr Infect Dis J 2009; 28:751-3. doi: 10.1097/INF.0b013e31819c6bfb.

16. Merchant S, Bharati A, Merchant N. Tuberculosis of the genitourinary system: Urinary tract tuberculosis - Renal tuberculosis: Part I. Indian J Radiol Imaging 2013; 23:46-63. doi: 10.4103/0971-3026.113615.

17. McDougal WS, Wein AJ, Kavoussi LR, Novick AC, Partin AW, Peters CA, et al. Campbell-Walsh Urology. 10th ed. Philadelphia, Pennsylvania, USA: Saunders, 2012. Pp. 1417-18.

18. Cantwell MF, Shehab ZM, Costello AM, Sands L, Green WF, Ewing EP, et al. Brief report: Congenital tuberculosis. N Engl Med 1994; 330:1051-4. doi: 10.1056/NEJM199404143301505.

19. Mazurek GH, Jereb J, Vernon A, LoBue P, Goldberg S, Castro K, et al. Updated guidelines for using interferon gamma release assays to detect Mycobacterium tuberculosis infection: United States, 2010. MMWR Recomm Rep 2010; 59:1-25.

20. Krishnamoorthy S, Gopalakrishnan G. Surgical management of renal tuberculosis. Indian J Urol 2008; 24:369-75. doi: 10.41 03/0970-1591.42620.

21. Gupta NP, Kumar R, Mundada OP, Aron M, Hemal AK, Dogra PN, et al. Reconstructive surgery for the management of genitourinary tuberculosis: A single center experience. J Urol 2006; 175:2150-4. doi: 10.1016/S0022-5347(06)00310-7. 